\title{
LAS AGUAS RESIDUALES URBANAS EN ANDALUCIA
}

\author{
Antonio CASTILLO MARTIN*
}

\section{INTRODUCCION}

Los vertidos de aguas residuales urbanas e industriales a las redes fluviales, al terreno o al mar, sin depurar o insuficientemente tratadas, plantean una grave problemática de reutilización (económica), sanitaria y ecológica, que es imprescindible afrontar con todos los medios a nuestro alcance. No hay que olvidar que dichos vertidos afectan directamente al recurso AGUA, eslabón primario y fundamental de la vida, y, consecuentemente, de todas las cadenas alimentarias. No creo necesario, por tanto, incidir en la trascendencia que supone alterar la calidad natural de dicho elemento. Ello cobra especial importancia, además, en Andalucía, región con graves y numerosos déficits hídricos, los cuales requieren de una más estricta gestión en cantidad y calidad de todas las aguas disponibles.

La problemática planteada por los vertidos de aguas residuales urbanas surge, más que de la potencialidad contaminante de dichos residuos líquidos, que no es alta en comparación con la de otros vertidos, industriales, mineros o agrícolas, de la extrema dispersividad de los puntos de emisión, así como de la heterogeneidad en cantidad y calidad de los mismos, lo que hace costosísimos los esfuerzos de depuración integral. Por otra parte, a pesar de que los costos de depuración son inferiores a los de vertidos de otras procedencias, el sustento de los mismos en comunidades urbanas normalmente inferiores a 50.000 habitantes es muy difícil de soportar por los propios ciudadanos.

En este artículo se expone, a grandes rasgos, la situación planteada por los vertidos de aguas residuales urbanas en Andalucía, al tiempo que se indican las grandes líneas de actuación, seguidas por otros países, necesarias para paliar la

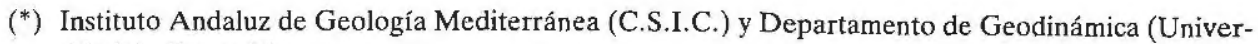
sidad de Granada). 
situación de grave deterioro que, en muchos casos, dichos vertidos están ocasionando en los sistemas hídricos andaluces. La información de base utilizada para la elaboración de este trabajo procede de las encuestas de sanidad ambiental(1), que fueron realizadas entre Octubre de 1983 y Febrero de 1985 por la Dirección General de Asistencia Primaria y Promoción de la Salud (Consejería de Salud y Consumo; Junta de Andalucía). Esta vasta información de base fue elaborada y parcialmente recogida para los 761 núcleos municipales de Andalucía en un trabajo titulado "Los vertidos de aguas residuales urbanas en Andalucía"(2), del cual se obtuvo una primera síntesis en otro trabajo titulado "Los vertidos de aguas residuales urbanas en Andalucía; problemática y valoración de los vertidos municipalizados"(3). Información global complementaria sobre el tema tratado puede obtenerse de los informes publicados por el Instituto Geológico y Minero de España o por la Dirección General de Obras Hidráulicas, entre otros.

\section{LOS VERTIDOS DE AGUAS RESIDUALES URBANAS EN ANDALUCIA}

El problema más grave de contaminación hídrica de Andalucía no es el originado únicamente por los vertidos de aguas residuales urbanas, las cuales constituyen, sin embargo, el foco de contaminación de carácter más regionalizado. En principio cabe considerar al estado de contaminación de nuestros ríos, embalses subterráneos y medio marino como originado por vertidos de diferentes procedencias, cuyos residuos interfieren en los naturales procesos de autodepuración y, consecuentemente, en el estado de calidad general de las aguas. No hay que olvidar, a este respecto, los vertidos de alpechín a nuestros ríos en la época de molturación de la aceituna, o los vertidos de importantes industrias situadas, sobre todo, en las márgenes del río Guadalquivir, que con sus vertidos, muchas veces tóxicos, inhiben o dificultan la natural actividad de depuración biológica. Así pues, hay que considerar a los vertidos de aguas residuales urbanas como un importante eslabón de la pesada cadena que supone el problema de la contaminación de las aguas, cuya depuración integral tendrá que afectar simultáneamente a los principales elementos que componen, en cada lugar, dicha cadena.

Andalucía, por su especial identidad y características, cuenta con una serie de condicionantes negativos de partida, cara a resolver la problemática de afección planteada por los vertidos de aguas residuales urbanas. En primer lugar, y como ya se apuntó en el epígrafe anterior, dispone de escasos recursos hídricos, lo que se traduce en ríos poco caudalosos, en los que pronto se superan las cargas contaminantes naturalmente autodepurables. Asimismo, el alto índice de dispersividad de la población, con sólo un 10,4 \% de núcleos urbanos que superan los 1.000 habitantes, unido a la configuración orográfica montañosa de una gran parte de 
Andalucía, dificultan en extremo las iniciativas de mancomunización y de agrupación de vertidos y de unidades de depuración. En contrapartida, la disponibilidad de litoral marino, en cuya franja costera se asienta el $25 \%$ de la población andaluza, facilita, en este caso, una evacuación relativamente facil y generalmente poco comprometida, a un medio como el marino que representa, en principio, una magnífica depuradora natural para los desechos de tipo orgánico.

Para plasmar en pocas palabras la situación de los vertidos de aguas residuales urbanas en Andalucía, baste decir que solo un $7 \%$ de los núcleos municipales cuentan con estaciones depuradoras operativas, las cuales agrupan a menos del $20 \%$ de la población total de Andalucía. En general, pues, los vertidos van a parar sin ningún tipo de tratamiento a los medios receptores, que, aproximadamente, son cauces fluviales en un $56 \%$, el mar en un $23 \%$ y el terreno en un $21 \%$.

Aún siendo preocupante la baja tasa de tratamiento de nuestros vertidos líquidos urbanos, es prioritario actuar sobre el estado de saneamiento de los nucleos urbanos de Andalucía. Todavía son frecuentes los nucleos que carecen de red de saneamiento, o la misma es insuficiente, lo que origina evacuaciones a fosas sépticas y, sobre todo, a pozos negros. Asimismo, cabe destacar el precario estado general de las conducciones en servicio, de edades medias altas y no construidas con los materiales adecuados; ello origina importantísimas pérdidas de los vertidos residuales en principio evacuados de las viviendas, cuando no se produce comunicación con las conducciones de abastecimiento.

El correcto diseño y construcción de las redes de saneamiento es, pues, uno de los problemas más graves con que se cuenta para planificar una eficaz depuración de las aguas residuales. Cuando la tendencia actual es la de construir redes separativas (aguas pluviales y de riego y aguas residuales), se siguen diseñando redes unitarias, que dificultan sobremanera el normal funcionamiento y restan rendimiento a las estaciones depuradoras. Cuando lo previsor sería intentar reunir los emisarios al máximo, para facilitar con ello la labor de una posterior depuración, los emisarios constituyen normalmente un rosario de salidas alrededor de los cascos urbanos; ello requerirá en un futuro de un ingente esfuerzo constructivo, y consecuentemente económico, por hacer canales de circunvalación que dirijan los vertidos hacia los mínimos puntos diferentes posibles.

Otro de los aspectos constructivos a resaltar es el de la ausencia o grave deficiencia de embovedados y colectores, lo cual permite que las aguas residuales urbanas discurran en muchos casos por el interior, o la inmediata proximidad, de los nucleos urbanos, desprendiendo malos olores y constituyendo un notable foco de contagios epidémicos; mención aparte merecen los colectores submarinos, todavía insuficientes y, generalmente, poco utilizados. Otro de los aspectos a resaltar es el alto índice relativo de utilización directa de aguas residuales urbanas en el riego de productos de huerta, lo que origina importantes riesgos sanitarios por consumo de verduras y hortalizas. 
A pesar de toda esta exposición, poco prometedora, de la situación de los vertidos de aguas residuales urbanas en Andalucía, no sería exacto ocultar que, en muchos casos, no se generan sensibles perjuicios a los medios receptores; ello se debe a que los vertidos son adecuadamente autodepurados. Sin embargo, es previsible pensar que dichas situaciones irán disminuyendo en un futuro, al aumentar los volumenes de vertido por unidades de cuenca, y, paralelamente, al hallarse mejor regulados y explotados los recursos hídricos, lo que finalmente originará incrementos de las cargas contaminantes, imposibles de asimilar en su totalidad por los medios receptores; es algo parecido a lo que ocurre estacionalmente en las épocas de molturación de la aceituna (vertidos de alpechín) y de estiaje, en las que muchos cauces se convierten en auténticas cloacas, pese a haberse disminuido notablemente el efecto de los vertidos de alpechín por las preceptivas balsas de decantación.

\section{CUANTIFICACION DE LOS VERTIDOS Y DISTRIBUCION ESPACIO-TEMPORAL}

Una cuantificación del volumen de vertidos de aguas residuales urbanas generado es imposible de realizar más que por aplicación de dotaciones medias de suministro por niveles de población. Ello representa, pues, solo una estimación aproximativa, según la cual los vertidos anuales son del orden de $450 \mathrm{Hm}^{3}$.

La repartición de este volumen de vertidos por cuencas hidrográficas y a nivel provincial aparece recogida en la tabla I. Tres son las unidades naturales principales receptoras de los vertidos generados, la cuenca del Guadalquivir (56\%), el mar $(23 \%)$ y las cuencas sur-Atlántica y Mediterránea (20\%). A este respecto, conviene resaltar que Andalucía cuenta con la gran ventaja, de cara a la gestión propia de los recursos hídricos, de coincidir en su práctica totalidad con unidades hidrográficas. Desde el punto de vista de una repartición provincial del volumen de vertidos generados, decir que se sigue, aproximadamente, la siguiente ordenación: Sevilla (24\%), Cádiz (16\%), Málaga (16\%), Granada (11\%), Córdoba $(11 \%)$, Jaén (10\%), Huelva (7\%) y Almería (5\%). Aunque escapa a la estadística reflejada en la tabla I, conviene resaltar la muy diferente distribución de los vertidos por unidades hidrográficas y en los medios receptores de unas provincias a otras (3). Entre las diferencias más notables, cabe señalar que para las provincias de Andalucía Oriental es mayor el porcentaje de reutilización de las aguas residuales urbanas (riegos) y de vertidos directos al suelo (pozos negros...); otra clara diferenciación es la de presentar una mayor atomización de los vertidos, producto de una mayor dispersividad de la población existente, que presenta su polo opuesto en la provincia de Cádiz, que con solo 42 nucleos municipales agrupa al $89 \%$ de su población total. 


\section{CUADRO I}

ESTADISTICA DE VERTIDOS DE AGUAS RESWUALES URBANAS DE LOS 761 NUCLEOS MUNICIPALES DE ANDALUCIA (1983-85)

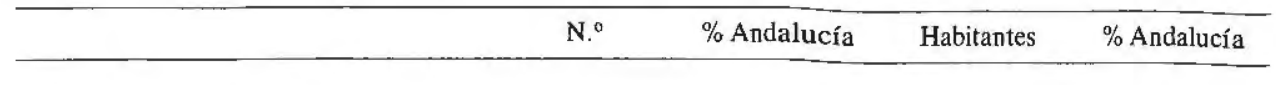

SUPERFICIE

87.277

\section{NUCLEOS URBANOS Y POBLACION}

Núcleos municipales

Anejos

Total de entidades

761
2.566
3.327

437

A cauces

Al suelo

21

Utilización regadio

Mixtos

Al mar

Mixtos

Sin red de saneamiento

-Distribución de vertidos

- Cuencas hidrográficas

Alto Guadalquivir

Medio Guadalquivir

Bajo Guadalquivir

Genil

Sur Mediterráneo

Sur Atlántica

Guadiana

Segura

Mar

- Provincias

Sevilla

Cádiz

Málaga

Granada

Córdoba

Jaén

Huelva

Almería
111

73

106

105

229

67

37

5

28

102

42

99

166

75

96

79

102

57,4
2,8
6,4
26,3
2,6
1,1
3,4

22,9

77,1

-

5.576 .839

864.918

6.441 .755

3,4

3.130 .290

27.107

114.038

986.217

1.004.437

277.447

37.303
86,6

13,4
00

20

8

26

56,1

0,5

2,0

17,7

18,0

5,0

0,7 
Una variación estacional del volumen de los vertidos no es apreciable por movimientos migratorios internos, aunque el efecto es notable por movimientos de población foránea en los meses de verano; esta "congestión" es especialmente importante en la zona turística de la Costa del Sol, donde la población estable es facilmente triplicada; este hecho ocasiona, como es de suponer, graves problemas de servicio, uno de los cuales es la evacuación de las aguas residuales urbanas, lo cual ocasiona, en muchos casos, problemas de contaminación local en playas de baño.

\section{MEDIOS RECEPTORES. PROBLEMATICAS DE DEPURACION Y DE VERTIDO}

Con un índice de tratamiento que apenas alcanza al $20 \%$ de los vertidos totales, puede decirse que el principal sistema de eliminación de los mismos es el de su desecho directo a los distintos medios receptores disponibles en cada caso.

Los valores reseñados en la tabla I, en la que se consideran los principales medios receptores de los vertidos, indican que el medio que soporta una mayor carga de vertidos directos son los cauces superficiales (ríos, arroyos, ramblas...), con un $56 \%$ de incidencia de vertidos únicos y un $18 \%$ de vertidos mixtos, a los que sigue el mar, con un $18 \%$ de vertidos únicos y un $5 \%$ de vertidos mixtos. Por fin, los vertidos directos al suelo (regadío, pozos negros...) presentan poca incidencia media, con solo un $3 \%$ de vertidos únicos y un $18 \%$ de vertidos mixtos, aunque como ya se apuntó en el epígrafe anterior, esta incidencia es notablemente mayor en las provincias de Andalucía Oriental, y, sobre todo, en las de Granada y Almería (10\% de vertidos únicos y 20-60\% de vertidos mixtos).

La problemática de afección planteada por los vertidos directos a los sistemas hídricos, y, en general, a la integridad del medio ambiente, se liga, lógicamente, al inexistente tratamiento de los mismos. Este hecho se halla fatalmente unido a un muy deficiente diseño, cobertura, construcción y mantenimiento de las redes de saneamiento urbanas existentes, sin olvidar, como problema principal, la dificultad de financiación y, sobre todo, de mantenimiento de las instalaciones de depuración correspondientes. La información disponible al respecto (1) establece un grado de disfuncionalidad de las estaciones construidas del $50 \%$, sin contar con el bajo rendimiento general de las instalaciones que permanecen operativas. Los motivos aducidos a la falta de operatividad y eficacias se concretan, básicamente, en graves dificultades de mantenimiento económico por parte de las corporaciones locales correspondientes y en problemas derivados del mantenimiento técnico de las instalaciones construidas. En cualquier caso, no cabe duda de 
que dichas razones, pese a ser ciertas, son frecuentemente explotadas como tapadera para eludir las cargas económicas que conlleva la responsabilidad ineludible de la depuración de las aguas; esta actitud se halla favorecida por el seguimiento permisivo de la legislación vigente.

Las alteraciones producidas en los medios receptores son de muy diferente grado según las específicas condiciones que concurren en cada caso concreto. No obstante, se puede admitir la generalidad de que los sistemas hídricos más afectados por los vertidos líquidos urbanos son los de superficie (arroyos, ríos, embalses...), seguidos de los subterráneos (acuíferos) y, por último, del medio marino.

Los sistemas hídricos de superficie y, en particular, los arroyos y ríos de poco caudal son, lógicamente, los más afectados en su integridad ecológica, que normalmente, sufre un cambio radical, ya que estos cauces de "cabecera" suelen presentar aguas totalmente puras hasta llegar a los puntos de emisión urbana. Esta problemática se ve favorecida por la "abusiva" derivación de aguas para regadio en verano. La afección producida en estos cursos superficiales se traslada inevitablemente a los embalses de la cuenca, que cada día presentan mayores índices de eutrofización.

Los embalses subterráneos, afectados por la infiltración directa (riegos, pozos negros, fugas...) e indirecta (ríos...) de las "aguas negras", presentan, en general, menores problemas de contaminación, hecho que se liga a sus mayores defensas naturales, ejercidas por la acción filtrante de la franja de terreno no saturada situada por encima del nivel piezométrico. Una casuística, lamentablemente frecuente, que origina numerosas contaminaciones de sistemas acuíferos es la de fugas e inyecciones de aguas residuales por pozos negros bajo el nivel piezométrico o en su inmediata proximidad.

En general, son poco frecuentes los casos de afección a sistemas acuíferos carbonatados (salvo por evacuaciones a simas o pozos negros), los cuales, sin embargo, presentan un escaso poder de autoprotección y rápidas velocidades de propagación del contaminante. Por el contrario, las afecciones a sistemas acuíferos detríticos son relativamente normales, ya que no hay que olvidar que muchos de ellos constituyen excelentes vegas, atravesadas e irrigadas por ríos y acequias que son frecuentemente cauce de evacuación de "aguas negras". En este sentido, el índice de contaminaciones microbiológicas de los puntos de abastecimiento que captan a dichos acuíferos es relativamente preocupante; a pesar de ello la problemática sanitaria más grave es la que se tiene planteada con la contaminación microbiológica por regadío de los productos de huerta. 
Por último, decir que el medio marino es el menos afectado por los vertidos líquidos urbanos, los cuales provocan, no obstante, importantes deterioros de uso (baño) en la franja litoral cercana a la localización de algunos emisarios submarinos. Se ha constatado experimentalmente que el medio marino tiene potencialidad suficiente para autodepurar y asimilar en sus cadenas alimentarias a los vertidos líquidos urbanos (afirmación no extensiva a los vertidos industriales tóxicos), aunque para ello requiera modificar la estructura ecológica natural de la zona de vertidos.

\section{POSIBILIDADES DE ACTUACION LINEAS PRIORITARIAS}

La problemática de vertidos planteada a nivel de Andalucía permite sugerir una serie de lineas prioritarias de actuación, las cuales, por otra parte, no suponen novedad ninguna, y responden y confirman, en general, los "decálogos" de actuación de las principales empresas y organismos encargados de redactar y supervisar los Planes Directores de saneamiento integral. Dichas recomendaciones serían las siguientes:

- Acometer las actuaciones de saneamiento de forma integrada (vertidos urbanos, industriales...), por unidades hidrográficas, y bajo la planificación y supervisión de un solo organismo competente y adecuadamente especializado.

- Centrar los esfuerzos prioritarios en el mejor diseño, cobertura y mantenimiento de las redes de saneamiento existentes, y en la instalación de estaciones de tratamiento en los núcleos urbanos interiores con poblaciones superiores a los 10.000 habitantes ( $50 \%$ de los vertidos totales). De forma paralela proceder también al tratamiento de aquellos vertidos urbanos que ocasionen graves trastornos al medio ambiente y/o a los potenciales usos ulteriores del agua.

- Dotar de instalaciones con tratamientos primarios y de emisarios submarinos, convenientemente diseñados y localizados, a las principales poblaciones costeras de interés turístico ( $23 \%$ de los vertidos). Alternativamente, promover, siempre que sea factible, la reutilización y recarga de acuíferos con dichos vertidos (pretratados), con el fin de atender a las altas demandas hídricas litorales y de dificultar los procesos de intrusión marina, que tan graves trastornos pueden ocasionar a la economía agraria. 
- Sustituir los riegos de productos hortícolas con aguas residuales urbanas por otros con aguas de aceptable calidad microbiológica.

- Incentivar la investigación, experimentación y desarrollo de sistemas de tratamiento de bajo coste de instalación y mantenimiento, adecuados a la climatología de Andalucía, que puedan constituir la solución al tratamiento de los vertidos urbanos de bajo volumen.

- Siempre que sea posible, favorecer la autodepuración controlada por el terreno de los vertidos (una vez desbastados) y proceder a la reutilización y recarga de acuíferos en aquellas zonas con importantes deficiencias hídricas.

- Articular las oportunas medidas de financiación, mantenimiento técnico y de formación de personal, que hagan factible la instalación y correcta conservación y uso de las estaciones de tratamiento correspondientes.

\section{BIBLIOGRAFIA CONSULTADA}

(1) CONSEJERIA DE SALUD Y CONSUMO. Junta de Andalucía (1985). Encuestas del Programa de Saneamiento Ambiental. Dirección General de Atención Primaria y Promoción de la Salud. Inéd. Sevilla.

(2) CASTILlO MARTIN, A. (1986). Los vertidos de aguas residuales urbanas en Andalucía (mapa de vertidos a escala 1:800.000 y memoria anexa). Ediciones ANEL. Granada.

(3) CASTILlO MARTIN, A. (1986). Los vertidos de aguas residuales urbanas en Andalucía. Problemática y valoración de los vertidos "municipalizados". II Simp. sobre el agua en Andalucía: I: 71-81. Granada. 